\title{
Now the drugs don't work: tamsulosin is ineffective as medical expulsive therapy
}

$\mathrm{M}$ edical expulsive therapy, in which agents that relax the ureter are administered to patients in order to ease the passage of small to moderate ureteral stones, has increasingly been used for the expectant management of patients with ureteral colic. Indeed, a-adrenoceptor antagonists, such as tamsulosin, are currently recommended in both the American Urological Association (AUA) and European Association of Urology (EAU) guidelines; however, these recommendations are supported by lowerlevel (2a) evidence only, from a variety of smaller studies and meta-analyses.

\section{4 ...medical expulsive therapy is not effective as an expectant management strategy... 77}

Now, results from the recently completed SUSPEND trial, published in The Lancet, conclusively prove that medical expulsive therapy with either tamsulosin or nifedipine is no more effective than placebo at increasing stone passage in patients with ureteral colic. Commenting upon the design of the trial, and reasons for the approach used, lead author Robert Pickard explains "The trial was powered on the basis of the smallest estimate of treatment effect, which was that the stone passage rate would be $10 \%$ higher for the tamsulosin group compared with that of the nifedipine group." When asked about the choice of outcome measures, Pickard adds "we chose the rate of need for further intervention at 4 weeks as our primary outcome measure. This measure was chosen as we considered it to be the outcome of most importance to patients, providers of care and health-care funders."

This study cohort included a total of 1,167 adult patients with at least one ureteral stone of $\leq 10 \mathrm{~mm}$ at the largest dimension at one of 24 treatment centres around the UK. Patients were randomly assigned to receive either tamsulosin, nifedipine or placebo as self-administered tablets for a period of 4 weeks. Each treatment group, consisting of 383 , 383 and 384 patients for tamsulosin, nifedipine or placebo, respectively, had similar baseline characteristics including age, stone size and location, duration of pain and general health.

At the end of the treatment period, study participants had rates of spontaneous stone passage of $81 \%, 80 \%$ and $80 \%$ in the tamsulosin, nifedipine and placebo groups, respectively (adjusted risk difference versus placebo: $1.3 \%$ for tamsulosin $[P=0.73]$ and $0.5 \%$ for nifedipine $[P=0.88])$. Furthermore, no significant differences in rates of spontaneous stone passage were detected between treatment groups after a follow-up duration of 12 weeks, nor were any significant differences observed in any of the secondary outcomes (including days of analgesic use and time to stone passage).

These results conclusively demonstrate that medical expulsive therapy is not effective as an expectant management strategy for patients with ureteral stones. This finding will prove controversial to many urologists as it directly contradicts the conclusions of many smaller clinical investigations and meta-analyses in this area. When asked to explain this divergence with previously published data, Pickard simply points out: "those interested in this subject will have to make judgements whether to give more weight to these previous trials showing benefit or to the results of our well-powered, methodologically sound trial carried out in a standard care setting, which showed no treatment effect."

This lack of agreement between the findings of this trial and other smaller studies highlights the importance of largescale randomized-controlled trials that are

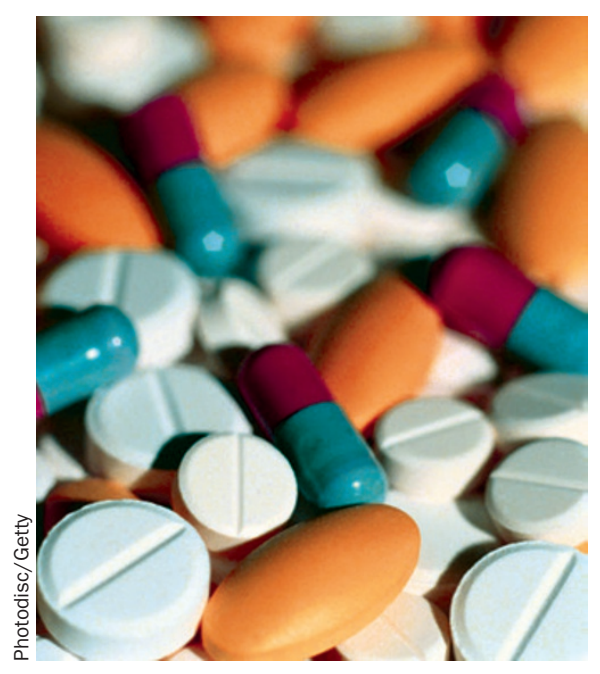

able to address areas of clinical uncertainty with sufficient statistical power. A further notable need exists for standardization of outcome measures amongst clinical studies in terms of the primary outcome measures used.

Summing up the most important message from this trial, Pickard states: "Patients, urologists and guideline writers will have to look at the results and decide whether these drugs should continue to be offered or used for this unlicensed indication."

Pickard goes on to add "We would like to highlight that such trials are a major undertaking and require the hard work of many people", although "without evidence from such trials we risk the continued use of treatments that are of uncertain or little benefit, to the detriment of patient care and resource usage."

\section{Peter Sidaway}

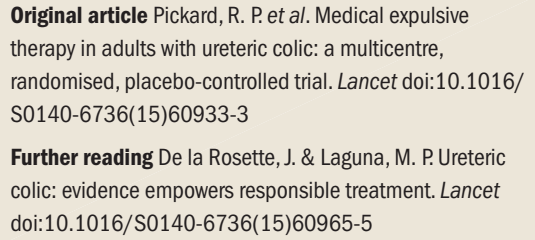

